\title{
Redução da Massa Molecular e Funcionalização do Poli(3-Hidroxibutirato-co-3-Hidroxivalerato) (PHBHV) Via Hidrólise Ácida e Transesterificação com Glicóis
}

\author{
Sérgio R. Montoro \\ Departamento de Engenharia de Materiais, Escola de Engenharia de Lorena, EEL - USP
}

\author{
Marli L. Tebaldi \\ Universidade Federal de Itajubá - UNIFEI, Campus de Itabira
}

\author{
Gizelda M. Alves, Jayne C. de S. Barboza \\ Departamento de Engenharia Química, EEL - USP
}

\begin{abstract}
Resumo: Neste trabalho foi realizado um estudo da redução da massa molecular do poli (3-hidroxibutirato-co-3-hidroxivalerato) (PHBHV) usando duas metodologias: hidrólise ácida com ácido clorídrico e transesterificação com etilenoglicol e hexilenoglicol. Foram investigados os parâmetros do processo: tempo, temperatura e concentração de catalisadores. Todas as metodologias estudadas geraram biopolímeros com massa molecular reduzida e funcionalizados com grupos hidroxila e carboxila terminais. Foram comparadas as metodologias estudadas, onde foi possível determinar a metodologia mais eficaz na funcionalização do PHBHV. A redução da massa molecular, associada a estratégias de funcionalização, é extremamente útil para promover alterações na taxa de degradação do PHBHV, ampliando assim as suas aplicações como biomateriais.
\end{abstract}

Palavras-chave: PHBHV, redução da massa molecular, funcionalização, hidrólise ácida, transesterificação.

\section{Reduction of Molecular Weight and Functionalization of Poly(3-Hydroxybutyrate-co-3-Hidroxyvalerate) (PHBHV) by Acid Hydrolysis and Transesterification with Glycols}

\begin{abstract}
In this work we investigated the reduction in molecular weight of poly(3-hydroxybutyrate-co-3-hydroxyvalerate) (PHBHV) with two methods: by acid hydrolysis and transesterification with ethylene and hexyleneglycol. The processing parameters studied were time, temperature and concentration of catalysts. Both methodologies resulted in functionalized biopolymers with hydroxyl and carboxyl end-groups and reduced molecular weight. A comparison of the methodologies allowed us to determine which one was more efficient for the functionalization of PHBHV. The reduction in molecular weight associated with functionalization strategies is extremely useful to induce changes in the degradation rate of materials, thus broadening their applications as biomaterials.
\end{abstract}

Keywords: PHBHV, molecular weight reduction, functionalization, acid hydrolysis, transesterification.

\section{Introdução}

Os primeiros polímeros utilizados em suturas bioabsorvíveis aprovadas na indústria médica começaram a ser produzidas a partir do ácido láctico e ácido glicólico em torno do ano de 1960. Desde então, outros dispositivos médicos, baseados em ácido láctico e ácido glicólico, bem como outros materiais, como o poli(trimetileno carbonato) e polímeros de poli( $\varepsilon$-caprolactama), foram aceitos como dispositivos médicos ${ }^{[1,2]}$.

Entre os polímeros biodegradáveis mais estudados nos últimos anos, especialmente para aplicações in vivo, estão os biopoliésteres bacterianos da família dos polihidroxialcanoatos (PHAs) ${ }^{[3,4]}$.

Os PHAs são polímeros sintetizados a partir de matérias primas renováveis pela agricultura, através de bactérias presentes em um meio nutritivo com excesso de fonte de carbono e podem ser degradados por microrganismos ${ }^{[5,6]}$. Além disso, devido à especificidade das enzimas microbianas, os polímeros resultantes apresentam regularidade estereoquímica e, consequentemente, possuem propriedades físicas reprodutíveis ${ }^{[7]}$. A biocompatibilidade e a baixa dissolução dos PHAs em ambientes biológicos (in vivo) os tornam úteis na medicina, na obtenção de materiais de sutura ${ }^{[8,9]}$, matrizes para liberação controlada de fármacos ${ }^{[10]}$ e também como materiais de suporte em fraturas ósseas, onde nesse caso, a propriedade piezoelétrica do polímero estimula o crescimento ósseo ${ }^{[11]}$. Além disso, esses materiais ainda podem ser utilizados, em algumas aplicações, como substitutos dos plásticos convencionais de origem petroquímica, como, por exemplo, em embalagens na indústria de alimentos ${ }^{[8,12]}$.

Entre os PHAs, o poli(3-hidroxibutirato), PHB, e o poli(3-hidroxibutirato-co-3-hidroxivalerato), $\mathrm{PHBHV}$, se enquadram entre os materiais mais atrativos para aplicações biomédicas. A utilização desses biopolímeros para implantes em tecidos já foi utilizada sem causar reações inflamatórias ou rejeição, além de, não resultar em produtos tóxicos para as células ${ }^{[13,14]}$. Por outro lado, apesar de possuírem características de biocompatibilidade, os polímeros de PHB são altamente cristalinos e termicamente instáveis na temperatura próxima ao seu ponto de fusão (em torno de $180{ }^{\circ} \mathrm{C}$ ). Essas propriedades intrínsecas do PHB influenciam nos mecanismos de degradação, distribuição e liberação do ativo, diminuindo seu potencial para algumas aplicações na área biomédica. A copolimerização do PHB com o 3-hidroxivalerato (3HV) é uma das estratégias promissoras para manipulação das 
propriedades térmicas e mecânicas desses materiais. A velocidade de degradação, bem como as propriedades termoplásticas podem ser ajustadas pela variação na composição monomérica dos seus comonômeros (HB e HV) e controle da massa molecular ${ }^{[15]}$.

Copolímeros de PHBHV possuem menor cristalinidade, maior maleabilidade e, consequentemente, maior velocidade de degradação, embora suas propriedades intrínsecas de hidrofobicidade podem restringir algumas aplicações in vivo. As superfícies do PHB e PHBHV são praticamente inertes e hidrofóbicas e não têm atividade fisiológica. Entretanto, sua citocompatibilidade pode ser melhorada através de modificações químicas com grupos funcionais ou modificações na superfície topográfica ${ }^{[16,17]}$. Essas modificações dos polímeros podem gerar interações mais favoráveis com as células permitindo ampliar seu potencial de aplicação como biomateriais.

Efetivas modificações químicas e diminuição da massa molecular dos polímeros podem ser obtidas através de processos de redução utilizando agentes redutores ou via hidrólise (ou alcoólise $)^{[16-22]}$. Renard et al. investigaram a influência da hidrólise no poli(3-hidroxibutirato-co-12\% 3-hidroxivalerato (PHBHV) e no poli(3-hidroxioctanoato) (PHO) na introdução de grupos polares, tais como grupos ácidos carboxílicos nas cadeias laterais. Os resultados mostraram que a presença de grupos carboxílicos nas cadeias laterais causou uma melhora significativa na hidrólise e na velocidade de degradação ${ }^{[17]}$. Copolímeros em blocos anfifílicos de baixa massa molecular (3000-30000) de PHB conjugados com o Poli(etilenoglicol) (PEG) foram obtidos através de reações de transesterificação ${ }^{[23]}$.

Baran et al. utilizaram a via de redução com borohidreto de sódio $\left(\mathrm{NaBH}_{4}\right)$ para a obtenção de polímeros de $\mathrm{PHBHV}$ de massa molecular reduzida, os quais foram utilizados para a preparação de nanocápsulas contendo proteínas usadas na terapia do câncer. Os testes realizados "in vitro" indicaram que os polímeros de baixa massa molecular apresentaram uma maior permeabilidade para solutos e, consequentemente, maior eficiência na atividade enzimática $^{[18]}$. Outros processos químicos para modificação de superfícies incluem, por exemplo, incorporação de unidades hidrofílicas via polimerização graft $^{[16]}$ ou tratamento com agentes oxidantes $^{[24]}$.

No Brasil, a empresa PHB Industrial S.A., vem desenvolvendo e comercializando PHAs sob a marca BIOCYCLE ${ }^{\circledR}$, a partir de fontes renováveis como a cana de açúcar e com a utilização de uma tecnologia limpa de produção. A produção é feita por meio da fermentação do açúcar da cana de açúcar e pelos microorganismos da espécie Alcalígenes sp. No processo de produção, o bagaço da cana de açúcar é usado como fonte de energia (elétrica e geração de vapor) e solventes não prejudiciais à saúde e ao meio ambiente são usados na extração dos biopolímeros. A iniciativa de produção desses PHAs no Brasil abre excelentes perspectivas de um maior desenvolvimento de sistemas de liberação controlada à base desses polímeros no país, especialmente devido ao menor custo, quando comparados aos poliésteres biodegradáveis derivados dos ácidos lático e glicólico, os quais por não serem produzidos no Brasil possuem maior valor agregado.

O propósito deste estudo foi a redução da massa molecular do PHBHV através de duas diferentes metodologias: hidrólise ácida utilizando-se ácido clorídrico $(\mathrm{HCl})$ em meio aquoso e a transesterificação com etilenoglicol ou hexilenoglicol catalisada por ácido $p$-toluenosulfônico. Diferentes parâmetros de processo foram investigados, tais como: tempo, temperatura e concentração de catalisadores. Os polímeros de PHBHV de massa molecular reduzida foram obtidos a fim de desenvolver sistemas carreadores de ativos mais eficientes quanto à permeabilidade e biodegrabilidade.

\section{Experimental}

\section{Materiais}

O PHA utilizado neste trabalho foi o poli(3-hidroxibutirato-co3-hidroxivalerato) (PHBHV), contendo 9,8\% de HV, cedido pela empresa PHB Industrial S/A $(\overline{\mathrm{Mw}}=273.204 \mathrm{Da}, \overline{\mathrm{Mn}}=192.028 \mathrm{Da}$, índice de polidispersão (IP) $=1,42$, temperatura de fusão $\cong 170{ }^{\circ} \mathrm{C}$ e grau de cristalinidade de aproximadamente $36 \%$ ). Clorofórmio PA (pureza $>99 \%$ ), piridina PA (pureza >99\%), álcool etílico PA (pureza 96\%) e ácido clorídrico PA (37\%), foram adquiridos da ANIDROL. O ácido $p$-toluenosulfônico foi fornecido pela empresa Tekno S/A. O etilenoglicol e hexilenoglicol foram fornecidos pela empresa Hexion Química. Todos os solventes e demais produtos químicos foram utilizados sem prévia purificação por apresentarem grau de pureza analítico.

\section{Procedimento geral}

A redução da massa molecular do PHBHV foi realizada através de duas metodologias: hidrólise ácida e transesterificação com alcoóis catalisadas por ácido.

\section{Redução da massa molecular via hidrólise ácida}

As reações de hidrólise ácida do PHBHV foram conduzidas nas temperaturas de 65,90 e $95{ }^{\circ} \mathrm{C}$, utilizando uma solução de ácido clorídrico $(3 \mathrm{~N})$ durante 12 horas. O mecanismo proposto para essas reações (Figura 1) sugere que, além do produto da degradação do PHBHV funcionalizado com uma hidroxila terminal, ocorre também a formação de uma estrutura insaturada.<smiles>CCC(CC(=O)C(C)(O)[In])OC(=O)CC(C)OC</smiles><smiles>CCOC(=O)c1ccc(CC(C)ONCCOC)o1</smiles><smiles>C/C=C/C(=O)OC(C)CC(=O)OC(CC)CC(=O)C(C)(C)O</smiles><smiles>CCC(CC(=O)O)OC(=O)CC(C)OC(C)(C)CC(=O)O</smiles>

Figura 1. Mecanismo proposto de hidrólise ácida do PHBHV (adaptado de Lauzier et al. ${ }^{[19]}$ ). 
Redução da massa molecular via transesterificação com etilenoglicol/hexilenoglicol

A redução da massa molecular do PHBHV foi efetuada a partir da reação de transesterificação com etilenoglicol e/ou hexilenoglicol, catalisada com ácido $p$-TS. Foram realizadas duas reações com diferentes quantidades de etilenoglicol e hexilenoglicol (10 e $20 \mathrm{~g}$ ) e diferentes quantidades de catalisador ( 3 e $6 \mathrm{~g}$ ). As reações foram realizadas na temperatura de $60{ }^{\circ} \mathrm{C}$ com duração de 3 horas.

A Figura 2 apresenta o mecanismo proposto para as reações de transesterificação, o qual se observa a formação de PHBHV funcionalizado.

\section{Purificação do PHBHV de baixa massa molecular}

Para a purificação dos polímeros obtidos via hidrólise ácida, o polímero foi filtrado e lavado com água destilada (3X) e a seguir com álcool etílico para a remoção dos traços de ácido residual. Após, os polímeros foram secos numa estufa de renovação e circulação de ar durante 24 horas na temperatura de $45^{\circ} \mathrm{C}$. Para os polímeros obtidos nas reações de transesterificação, foi adicionada uma solução de bicarbonato de sódio $\left(\mathrm{NaHCO}_{3} 10 \%\right)$. A fase orgânica foi separada, o polímero precipitado em álcool etílico gelado e seco nas mesmas condições descritas anteriormente.

\section{Caracterização}

\section{Cromatografia de Permeação em Gel (GPC)}

As massas moleculares médias e as distribuições das massas moleculares dos polímeros foram determinadas por GPC/SEC, em um equipamento Waters Breeze System, incluindo uma bomba isocrática, Waters 1515, injetor automático, Waters 717 Plus e detector de índice de refração, Waters 2414. Foram utilizadas três colunas Styragel HR com faixa de exclusão de 500-30.000 (HR3), 5.000-600.000 (HR4), e 50.000-4.000.000 (HR5). As amostras de PHBHV foram diluídas na razão de $15 \mathrm{mg}$ por $1,0 \mathrm{~mL}$ de clorofórmio grau espectroscópico (J. T. Baker) e depois mantidas em repouso durante 20 horas para garantir a total dissolução do polímero. Posteriormente, as soluções foram filtradas com filtro de PTFE, com diâmetro de poro $0,45 \mu \mathrm{m}$ (Millex LCR). O volume de injeção foi de $100 \mu \mathrm{L}$, sob fluxo constante de $1,0 \mathrm{~mL} / \mathrm{min}$ do eluente na temperatura de $30{ }^{\circ} \mathrm{C}$. Os padrões usados no método de calibração universal foram os de poliestireno (PS) (desvio experimental máximo de 10\%).

Espectroscopia no Infravermelho com Transformada de Fourier (FTIR)

As análises de infravermelho foram efetuadas em um equipamento Perkin-Elmer, modelo Spectrum One. Os polímeros foram analisados no modo de transmitância, num comprimento de onda de 500 a $4100 \vee\left(\mathrm{cm}^{-1}\right)$. Todas as análises foram realizadas utilizandose as amostras de menor valor de massa molecular para facilitar a identificação do grupamento hidroxila.

\section{Ressonância Magnética Nuclear $\left(R M N^{1} H\right)$}

As análises de $\mathrm{RMN}^{1} \mathrm{H}$ foram efetuadas em um equipamento Varian Oxford, $300 \mathrm{MHz}$, usando como solvente clorofórmio deuterado $\left(\mathrm{CDCl}_{3}\right)$.

\section{Dosagens de grupos hidroxilas}

A concentração de grupos hidroxila, presentes nas extremidades das cadeias de PHBHV, antes e após a redução da massa molecular, foi obtida usando a técnica de titulação em meio orgânico, utilizando solução acilante e indicador de coloração, conforme metodologia adaptada da literatura ${ }^{[22,25]}$. PHBHV $(0,20 \mathrm{~g})$ foram solubilizados em $25 \mathrm{~mL}$ de solução de piridina em anidrido ftálico $\left(850 \pm 10 \mathrm{mMol} . \mathrm{L}^{-1}\right)$. Após a total dissolução, a mistura reacional foi deixada em refluxo por 75 minutos para promover a acetilação.

A seguir, o sistema foi resfriado em banho de gelo, adicionado $20 \mathrm{~mL}$ de piridina e $50 \mathrm{~mL}$ de água destilada. Uma solução de hidróxido de sódio $1 \mathrm{M}(\mathrm{NaOH} 1 \mathrm{M})$ foi usada para titular a mistura de reação e fenolftaleína foi usada como indicador de coloração.

A prova em branco foi feita para determinar a acidez residual da mistura acilante (anidrido ftálico em piridina). Todas as titulações para a determinação dos grupos hidroxila foram feitas em duplicata. A concentração dos grupos hidroxila presentes nas extremidades da cadeia polimérica do PHBHV foi quantificada utilizando-se as Equações 1 e 2:

$$
\begin{aligned}
& I O H=\frac{\left(V_{A}-V_{B}\right) \cdot 56,1 . f}{m} \\
& \% O H=\frac{I O H \cdot 1700}{56100}
\end{aligned}
$$

onde:

- $I O H=$ índice de grupos hidroxila (mg KOH / g de polímero)

- $V_{B}=$ volume gasto de $\mathrm{NaOH} 1 \mathrm{M}$ na prova branca $(\mathrm{mL})$;

- $V_{A}=$ volume gasto de $\mathrm{NaOH} 1 \mathrm{M}$ na amostra contendo polímero $(\mathrm{mL})$

- $m=$ massa da amostra (g);

- $f=$ fator de correção da solução de $\mathrm{NaOH} 1 \mathrm{M}$;

- 56,1 = equivalente-grama do $\mathrm{KOH}\left(\mathrm{g} \cdot \mathrm{mol}^{-1}\right)$.

Dosagens dos grupos carboxílicos

Para determinar a concentração de grupos carboxílicos nas extremidades das cadeias de PHBHV, antes e após a redução da massa molecular, foi utilizado a metodologia já descrita na<smiles>CCC(CC(=O)C(C)(C)O)OC(=O)CC(C)OC</smiles><smiles>C1C2CC1C2</smiles><smiles>OCCO</smiles><smiles>CCC(CC(=O)C(C)(C)OCO)OC(=O)CC(C)OC</smiles><smiles>CCC(CC(=O)OCCO)OC(=O)CC(C)OC</smiles>

Figura 2. Transesterificação via catálise ácida do PHBHV com etilenoglicol e hexilenoglicol. 
Tabela 1. Valores de $\overline{\mathrm{Mn}}, \overline{\mathrm{Mw}}$ e IP das reações de hidrólise ácida com $\mathrm{HCl} 3 \mathrm{~N}$ nas temperaturas de 65,90 e $95{ }^{\circ} \mathrm{C}$.

\begin{tabular}{|c|c|c|c|c|c|c|c|c|c|}
\hline \multirow{2}{*}{$\begin{array}{l}\text { Tempo } \\
\text { (horas) }\end{array}$} & \multicolumn{3}{|c|}{$\operatorname{ReHA}[1] 65^{\circ} \mathrm{C}$} & \multicolumn{3}{|c|}{ ReHA[2] $90^{\circ} \mathrm{C}$} & \multicolumn{3}{|c|}{ ReHA[3] $95{ }^{\circ} \mathrm{C}$} \\
\hline & $\overline{\mathrm{Mn}}(\mathrm{Da})$ & $\overline{\mathrm{Mw}}$ (Da) & IP & $\overline{\mathrm{Mn}}(\mathrm{Da})$ & $\overline{\mathrm{Mw}}$ (Da) & IP & $\overline{\mathrm{Mn}}(\mathrm{Da})$ & $\overline{\mathrm{Mw}}$ (Da) & IP \\
\hline 0 & 192028 & 273204 & 1,42 & 192028 & 273204 & 1,42 & 192028 & 273204 & 1,42 \\
\hline 2,0 & 117265 & 187926 & 1,60 & 39474 & 80401 & 2,04 & 21032 & 84566 & 4,02 \\
\hline 4,0 & 82610 & 156022 & 1,89 & 25949 & 38742 & 1,49 & 10666 & 23584 & 2,21 \\
\hline 6,0 & 75299 & 126102 & 1,67 & 16813 & 34945 & 2,08 & 8623 & 17460 & 2,02 \\
\hline 8,0 & 73330 & 117226 & 1,60 & 12574 & 26361 & 2,10 & 6306 & 14193 & 2,25 \\
\hline 10,0 & 59278 & 98137 & 1,66 & 11760 & 24218 & 2,06 & 8001 & 13667 & 1,71 \\
\hline 12,0 & 51790 & 82776 & 1,60 & 9800 & 19518 & 1,99 & 6366 & 12307 & 1,93 \\
\hline
\end{tabular}

literatura $^{[26]}$. PHBHV $(0,20 \mathrm{~g})$ foram solubilizados em $25 \mathrm{~mL}$ de piridina PA e a mistura foi aquecida até a total dissolução. Após a dissolução, o sistema foi resfriado em banho de gelo. Uma solução alcoólica de hidróxido de sódio 0,1M em metanol $(\mathrm{NaOH} / \mathrm{MeOH}$ $0,1 \mathrm{M}$ ) foi usada para titular a mistura, e azul de bromo timol foi usado como indicador de coloração. Todas as titulações foram feitas em duplicata, e a média dos valores encontrados foi utilizada para os cálculos. A concentração dos grupos carboxílicos presentes nas extremidades da cadeia polimérica do PHBHV foi quantificada utilizando-se a Equação 3:

$$
[\mathrm{COOH}]=\frac{V_{\mathrm{NaOH}} \cdot f_{\mathrm{NaOH}} \cdot M_{\mathrm{NaOH}}}{1000 \cdot m_{\text {polimero }}}
$$

Onde:

- $[\mathrm{COOH}]=$ concentração de grupos $\mathrm{COOH}\left(\mathrm{mol} . \mathrm{g}^{-1}\right)$;

- $V_{\mathrm{NaOH}}=$ volume gasto de $\mathrm{NaOH} / \mathrm{MOH}(\mathrm{L})$;

- $f_{\mathrm{NaOH}}=$ fator de correção da solução de $\mathrm{NaOH} / \mathrm{MOH}$;

- $M_{\mathrm{NaOH}}=$ concentração da solução de $\mathrm{NaOH} / \mathrm{MOH}\left(\mathrm{mol} . \mathrm{L}^{-1}\right)$;

- $m_{\text {polimero }}=$ massa de polímero $(\mathrm{g})$.

\section{Resultados e Discussão}

\section{Hidrólise ácida}

A reações de hidrólise ácida do PHBHV foram realizadas nas temperaturas de 65,90 e $95{ }^{\circ} \mathrm{C}$, empregando uma solução de $\mathrm{HCl} 3 \mathrm{~N}$. Os resultados obtidos através das análises de GPC para a redução da massa molecular do PHBHV nas três temperaturas estão apresentados na Tabela 1. Esses resultados indicaram que a redução da massa molecular apresentou dois estágios de velocidade: nas primeiras 4 horas a massa molecular foi reduzida em torno de 20 vezes (temperatura de $95{ }^{\circ} \mathrm{C}$ ). O segundo estágio ( 8 horas) foi caracterizado por uma menor taxa de redução de massa molecular, tornando-se quase linear com o decorrer do tempo. Nesse período a massa molecular sofreu uma redução inferior a duas vezes.

Analisando os perfis da redução da massa molecular em função das três temperaturas estudadas, observou-se que, quanto maior a temperatura reacional maior a velocidade inicial de redução, ou seja, maior a diferença entre os dois estágios característicos da redução de massa molecular. Esse comportamento é devido à cisão aleatória das ligações éster do PHBHV, o qual já havia sido observado por Lauzier et al. ${ }^{[19]}$. No trabalho de Montoro et al. foram avaliados os resultados de redução da massa molecular ponderal média relativa à massa molar inicial do $\mathrm{PHBHV}, \mathrm{M}_{\mathrm{p}}(\mathrm{t}) / \mathrm{M}_{\mathrm{p} 0}$ nas temperaturas de 65,90 e $95^{\circ} \mathrm{C}$ do processo de hidrólise ácida.

Os autores apresentaram curvas de grau de conversão isotérmico, $\alpha$ em função do tempo, onde foi claramente observado que a $65^{\circ} \mathrm{C}$, a cinética de reação é significativamente mais lenta do que nas demais temperaturas ${ }^{[27]}$.

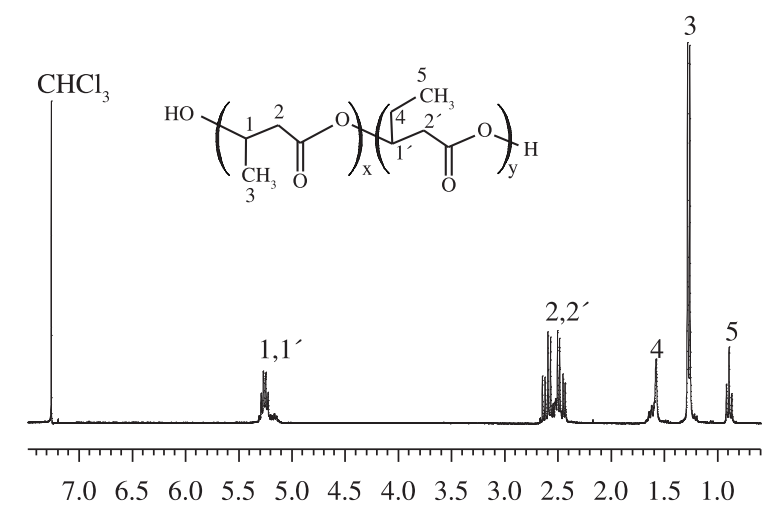

(a)

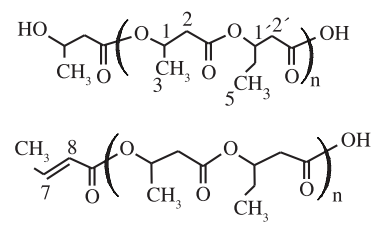

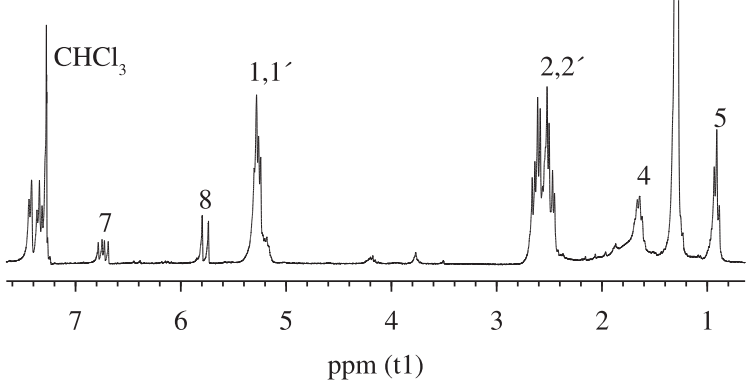

(b)

Figura 3. Espectro de $\mathrm{RMN}^{1} \mathrm{H}$ do PHBHV de partida (a) e do produto obtido da alíquota da reação ReHA[3] após 12 horas de reação (b).

Através de análises de $\mathrm{RMN}^{1} \mathrm{H}$ (Figura 3) observou-se a formação de subprodutos insaturados decorrentes da degradação térmica associada à hidrólise ácida. Tal comportamento foi observado nos trabalhos de Yu et al., no qual os autores sugerem que a formação desses subprodutos é devido à desidratação a partir de uma hidroxila final da cadeia do $\mathrm{PHBHV}, \mathrm{OH}-\mathrm{CH}\left(\mathrm{CH}_{3}\right)-\mathrm{CH}_{2}$ - COO -, seguido de uma $\beta$-eliminação após a quebra das cadeias a partir da hidrólise ${ }^{[24,28]}$.

\section{Transesterificação com glicóis}

A preparação de polímeros de PHBHV de massa molecular reduzida e funcionalizados com terminações hidroxiladas via transesterificação com etilenoglicol já havia sido estudada por 
Hirt et al. ${ }^{[29]}$. No presente trabalho as reações de transesterificação foram realizadas comparando-se dois glicóis (etilenoglicol e hexilenoglicol) e catalisadas pelo ácido p-toluenosulfônico. A presença do catalisador induz a cisão da ligação éster do PHBHV inicial, seguida da esterificação, originando um produto de cadeia final dihidroxilado. A Tabela 2 apresenta os valores de $\overline{\mathrm{Mn}}, \overline{\mathrm{Mw}}$ e IP obtidos através de GPC para as reações de transesterificação com etilenoglicol e hexilenoglicol. Semelhantemente às reações de hidrólise ácida, as transesterificações também ocorreram de acordo com um mecanismo de cisão aleatória, uma vez que a massa molecular do produto degradado decresceu rapidamente no início da reação. Para as reações realizadas em hexilenoglicol, foram obtidos polímeros finais com massas moleculares menores quando comparados aos polímeros obtidos nas reações com etilenoglicol. Isso pode ser atribuído à melhor solubilidade do hexilenoglicol no meio orgânico. As distribuições das massas moleculares foram ligeiramente mais estreitas para o sistema conduzido com hexilenoglicol. No entanto, as diferenças não foram significativas e os valores mantiveram-se praticamente constantes no decorrer das reações de transesterificação.

Tabela 2. Valores de $\overline{\mathrm{Mn}}, \overline{\mathrm{Mw}}$ e IP obtidos pela técnica de Cromatografia de Permeação em Gel (GPC) das alíquotas retiradas no decorrer da reação de transesterificação do PHBHV com etilenoglicol e hexilenoglicol, catalisada por ác. $\rho$-TS a $60{ }^{\circ} \mathrm{C}$.

\begin{tabular}{|c|c|c|c|c|c|c|}
\hline \multicolumn{7}{|c|}{ Redução via transesterificação com etilenoglicol } \\
\hline \multirow{2}{*}{$\begin{array}{l}\text { Tempo } \\
\text { (horas) }\end{array}$} & \multicolumn{3}{|c|}{ ReTEG[1] } & \multicolumn{3}{|c|}{ ReTEG[2] } \\
\hline & $\overline{\mathrm{Mn}}(\mathrm{Da})$ & $\overline{\mathrm{Mw}}(\mathrm{Da})$ & IP & $\overline{\mathrm{Mn}}(\mathrm{Da})$ & $\overline{\mathrm{Mw}}(\mathbf{D a})$ & IP \\
\hline 0 & 192028 & 273404 & 1,42 & 192028 & 273404 & 1,42 \\
\hline 1,0 & 45360 & 87948 & 1,94 & 47872 & 83994 & 1,75 \\
\hline 2,0 & 33613 & 62532 & 1,86 & 30848 & 53578 & 1,74 \\
\hline 3,0 & 16042 & 30113 & 1,88 & 27038 & 44092 & 1,63 \\
\hline \multicolumn{7}{|c|}{ Redução via transesterificação com hexilenoglicol } \\
\hline \multirow{2}{*}{$\begin{array}{l}\text { Tempo } \\
\text { (horas) }\end{array}$} & \multicolumn{3}{|c|}{ ReTHG[1] } & \multicolumn{3}{|c|}{ ReTHG[2] } \\
\hline & $\overline{\mathrm{Mn}}(\mathrm{Da})$ & $\overline{\mathrm{Mw}}(\mathrm{Da})$ & IP & $\overline{\mathrm{Mn}}(\mathrm{Da})$ & $\overline{\mathrm{Mw}}(\mathrm{Da})$ & IP \\
\hline 0 & 192028 & 273404 & 1,42 & 192028 & 273404 & 1,42 \\
\hline 1,0 & 19349 & 28490 & 1,47 & 20524 & 31863 & 1,55 \\
\hline 2,0 & 18164 & 26602 & 1,46 & 10236 & 15544 & 1,52 \\
\hline 3,0 & 7692 & 12783 & 1,66 & 8184 & 12255 & 1,50 \\
\hline
\end{tabular}

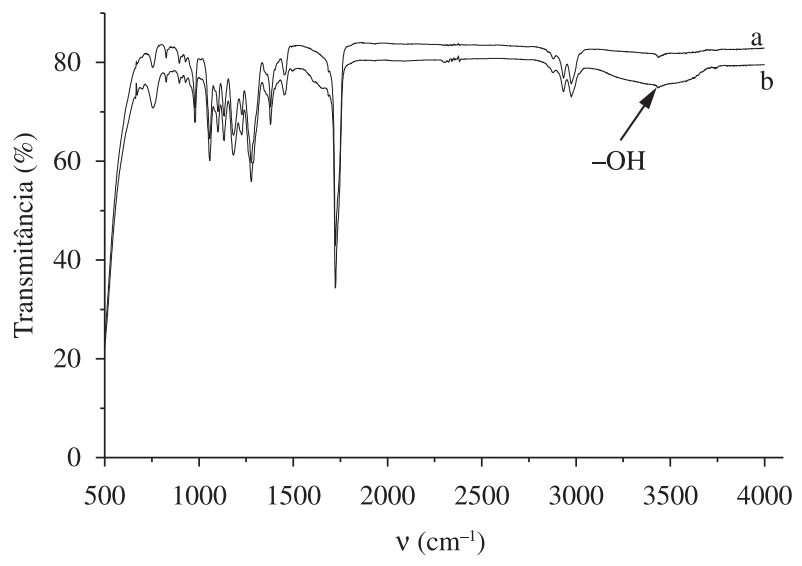

a: PHBHV partida b: ReHA[3] - 12 horas

(a)

\section{Comparação entre hidrólise ácida e transesterificação}

A redução da massa molecular do PHBHV pode ser eficientemente conduzida tanto via hidrólise ácida como via transesterificação com glicóis. No entanto, a via de redução por meio da transesterificação apresentou uma maior velocidade de redução da massa molecular, comparada com a hidrólise ácida. A observação e análises dos resultados de redução da massa molecular do PHBHV mostrou que foi possível obter polímeros com valores de massa molecular ponderada $(\overline{\mathrm{Mw}})$ na faixa de $12 \mathrm{KDa}$, através das reações de transesterificação após 3 horas de reação, na temperatura de $60{ }^{\circ} \mathrm{C}$. Por outro lado, a obtenção de polímeros na mesma faixa de massa molecular $(12 \mathrm{KDa})$ através das reações de hidrólise ácida, só foi alcançada após 12 horas de reação na temperatura de $95^{\circ} \mathrm{C}$. Os resultados estão de acordo com o trabalho realizado pelo nosso grupo de pesquisa, no qual foi observado que as reações de redução da massa molecular via hidrólise ácida foram mais lentas do que as reações de transesterificação, uma vez que as constantes de reação ( $k$ ) das reações de hidrólise ácida foram menores, comparados com os valores obtidos nas reações de transesterificação ${ }^{[27]}$.

Comparando-se os espectros de FTIR (Figura 4) do PHBHV de partida (a) com o espectro da reação ReHA[3] (b) da Figura 4a, pode ser observada uma banda de absorção na faixa em torno de 3100 a $3600 \mathrm{~cm}^{-1}$, característica do grupamento hidroxila, fato que comprova a funcionalização conforme o mecanismo proposto de hidrólise ácida do PHBHV. Na Figura 4b, a qual mostra o espectro do PHBHV de partida (a) e os espectros obtidos após as reações de transesterificação ReTEG[1] (b) e ReTHG[2] (c), também foi possível observar claramente a presença da banda de absorção do grupamento hidroxila $\left(v=3100\right.$ a $\left.3600 \mathrm{~cm}^{-1}\right)$. Isso comprova de forma qualitativa, que para ambos os mecanismos de redução da massa molecular estudados nesse trabalho, os produtos obtidos apresentaram o grupamento hidroxila na extremidade da cadeia polimérica do PHBHV de baixa massa molecular.

\section{Dosagens dos grupos hidroxilas $(-\mathrm{OH})$ e dos grupos carboxílicos $(-\mathrm{COOH})$}

Para a dosagem dos índices de hidroxilas (IOH), porcentagem de hidroxilas $(\% \mathrm{OH})$ e da concentração de grupos carboxílicos $([\mathrm{COOH}])$, foram utilizadas amostras de menor massa molecular

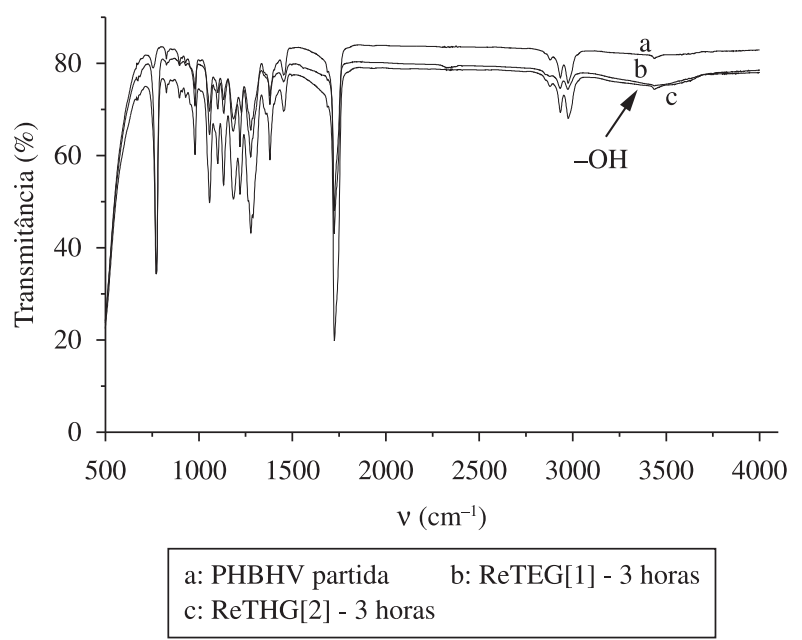

(b)

Figura 4. Espectros de FTIR do PHBHV das reações ReHA[3] no tempo de 12 horas (a) e do PHBHV das reações ReTEG[1] e ReTHG[2] no tempo de 3 horas (b), obtidos em filmes sob pastilha de $\mathrm{NaCl}$. 
Tabela 3. Resultados do índice de hidroxilas (IOH), da porcentagem de hidroxilas $(\% \mathrm{OH})$ e da concentração de grupos carboxílicos ([COOH]) da alíquota da reação ReHA[3] no tempo igual a 12 horas e das alíquotas das reações ReTEG[1] e ReTHG[2] no tempo igual a 3 horas.

\begin{tabular}{cccc}
\hline Alíquotas & $\begin{array}{c}\mathbf{I O H} \\
(\mathbf{m g} \mathbf{d e} \mathbf{K O H} / \mathbf{g} \\
\mathbf{d e ~ P H B H V})\end{array}$ & $\begin{array}{c}\text { Porcentagem de } \\
\text { grupos } \mathbf{~ O H} \\
(\%)\end{array}$ & $\begin{array}{c}{[\mathbf{C O O H}]} \\
\left(\mathbf{m o l} . \mathbf{g}^{-1}\right)\end{array}$ \\
\hline $\begin{array}{c}\text { ReHA[3] } \\
12 \text { horas }\end{array}$ & 53,79 & 1,63 & $6,82 \times 10^{-7}$ \\
$\begin{array}{c}\text { ReTEG[1] } \\
\text { 3 horas }\end{array}$ & 77,88 & 2,36 & $6,76 \times 10^{-7}$ \\
$\begin{array}{c}\text { ReTHG[2] } \\
\text { 3 horas }\end{array}$ & 79,86 & 2,42 & $6,95 \times 10^{-7}$ \\
\hline
\end{tabular}

para propiciar a detecção dos grupos funcionais presentes na extremidade da cadeia polimérica do PHBHV obtidos pelas duas metodologias estudadas nesse trabalho. A Tabela 3 apresenta os resultados obtidos por meio da técnica de titulação em meio orgânico, os quais comprovaram, de maneira quantitativa, a funcionalização do $\mathrm{PHBHV}$ por grupos $-\mathrm{OH}$ e $-\mathrm{COOH}$ na extremidade da cadeia do PHBHV.

Nas análises de $\mathrm{RMN}^{1} \mathrm{H}$ das amostras de PHBHV obtidos via transesterificação, não foi possível identificar os sinais correspondentes dos polímeros funcionalizados. Esse comportamento é comum e pode ser explicado devido aos valores de massa molecular dos polímeros resultantes não serem suficientemente baixos para serem detectados através das análises de $\mathrm{RMN}^{1} \mathrm{H}$.

\section{Conclusões}

A redução da massa molecular do PHBHV ocorreu de maneira satisfatória e o copolímero de baixa massa molecular gerado foi funcionalizado com a inserção de grupos funcionais $-\mathrm{OH}$ e $-\mathrm{COOH}$ nas extremidades das cadeias poliméricas. A redução da massa molecular do PHBHV por meio do mecanismo de cisão de cadeia aleatória foi favorecida com o aumento da temperatura da reação de hidrólise. A transesterificação com hexilenoglicol foi o processo de redução que proporcionou uma maior quantidade de cadeias finais funcionalizadas com grupos hidroxila no PHBHV. Além disso, os polímeros resultantes apresentaram uma melhor distribuição de massas moleculares (IP), quando comparado com a hidrólise ácida.

A redução de massa molecular associada à funcionalização são estratégias extremamente úteis para ocasionar mudanças na velocidade de degradação dos materiais, ampliando assim as aplicações como biomateriais.

\section{Agradecimentos}

Os autores agradecem a empresa PHB Industrial S.A., pela doação de todo o PHBHV utilizado neste trabalho e pela execução das análises de GPC e ao CNPq e à FAPESP pelo apoio financeiro.

\section{Referências Bibliográficas}

1. Kumar, N.; Langer, R. S. \& Domb, A. J. - Adv. Drug Delivery Rev., 54, p.889 (2002).

2. Domb, A. J.; Amselem, S.; Langer, R. - Maniar, M. - in: "Polyanhydrides as carriers of drugs", S. Shalaby (Ed.), Designed to Degrade Biomedical Polymers, Hanser, Munich, p.69 (1994).
3. Tang, C. Y.; Chen, D. Z.; Yue, T. M.; Chan, K. C.; Tsui, C. P. \& Yu, H. F. - Comp. Sci. Technol., 68, p.1927 (2008).

4. Misra, S. K.; Valappil, S. P.; Roy, I. \& Boccaccini, A. R. Biomacromolecules, 7, p.2249 (2006).

5. Rocha, R. C. S.; Pereira, F. S.; Taciro, M. K.; Rodrigues, M. F. A. \& Pradella, J. G. C. - Quim. Nova, 30, p.53 (2007).

6. Du, G. C.; Chen, J.; Yu, J. \& Lun, S. - Biochemi. Eng. J., 8, p.103 (2000).

7. Pouton, C. W. \& Akhtar, S. - Adv. Drug Delivery Rev., 18, p.133 (1996).

8. Hrabak, O. - FEMS Microbiol. Rev., 103, p.251 (1992).

9. Steinbüchel, A. - Chem. Labor Biotech., 44, p.378 (1993).

10. Franchetti, S. M. M. \& Marconato, J. C. - Quim. Nova, 29, p.811 (2006).

11. Fialho, S. L.; Rego, M. G. B.; Cardilho, J. A.; Siqueira, R. C.; Jorge, R. \& Cunha Júnior, A. S. - Arq. Bras. Oftalmol., 66, p.891 (2003).

12. Kunze, C.; Freier, T.; Kramer, S. \& Schmitz, K. P. J. - Mat. Sci.: Mat. Med., 13, p.1051 (2002).

13. Laffel, L. - Diabetes Metab. Res. Rev., 15, p.412 (1999).

14. Steinbüchel, A. - in: "PHB and other Polyhydroxyalkanoic acids", H. J. Rehm, G. Reed, M. Roehr (Ed.), 2. ed., Willey: John \& Sons, p.405 (1996).

15. Pouton, C. W. \& Akhtar, S. - Adv. Drug Delivery Rev., 18, p.133 (1996).

16. Lao, H.; Renard, E.; Linossier, I.; Langlois, V. \& Vallée-Rehel, K. Biomacromolecules, 8, p.416 (2007).

17. Renard, E.; Walls, M.; Guerina, P. \& Langlois, V. - Polym. Degrad. Stab., 85, p.779 (2004).

18. Baran, E. T.; Oézer, N. \& Hasirci, V. J. - Microencapsulation, 19, p.363 (2002).

19. Lauzier, C.; Revol, J. - F.; Debzi, E. M. \& Marchessault, R. H. Polymer, 35, p.4156 (1994).

20. Yu, G. \& Marchessault, R. H. - Polymer, 41, p.1087 (2000).

21. Spitalsky, Z.; Lacík, I.; Lathová, E.; Janigová, I. \& Chodák, I. - Polym. Degrad. Stab., 91, p.856 (2006).

22. Montoro, S. R. - "Redução da Massa Molar do Poli(3-Hidroxibutiratoco-3-Hidroxivalerato) (PHBHV) para sua Posterior Utilização no Desenvolvimento de Sistemas de Liberação Controlada", Dissertação de Mestrado, Universidade de São Paulo, Brasil (2005).

23. Jeong, K. H. \& Young, J. K. - Polymer, 30, p.512 (2006).

24. Yu, D. G.; Lin, Y. C. \& Yang, M. C. - Macromol. Biosci., 6, p.348 (2006).

25. Deng, X. M.; Hao, J. Y. - Eur. Polym. J., 37, p.211 (2001).

26. Oliveira, M. P.; Giordani, D. S. \& Santos, A. M. - Eur. Polym. J., 42, p.1196 (2006).

27. Montoro, S. R.; Shigue, C. Y.; Sordi, M. L T.; Santos, A. M. \& Ré, M. I. - Polímeros, 20, p.19 (2010).

28. Yu, G. \& Marchessault, R. H. - Polymer, 41, p.1087 (2000).

29. Hirt, T. D.; Neuenschwander, P.; Suter, U. W. - Macromol. Chem. Phys., 197, p.1609 (1996).

Enviado: $31 / 07 / 10$

Reenviado: $22 / 09 / 10$

Aceito: $27 / 10 / 10$

DOI: $10.1590 / \mathrm{S} 0104-14282011005000044$ 